\title{
Comparison of clinical efficacy of oxcarbazepine and lamotrigine combined with escitalopram, and impact on prognostic quality of life in treating patients with epilepsy and depressive disorder
}

\author{
XIAOGUANG $\mathrm{ZHANG}^{1}$ and WENLI ZHAO ${ }^{2}$ \\ ${ }^{1}$ Center of Brain Diseases, Sunshine Union Hospital, Weifang, Shandong 261061; \\ ${ }^{2}$ Department of Neurosurgery, Weifang No. 2 People's Hospital, Weifang, Shandong 261041, P.R. China
}

Received July 11, 2019; Accepted December 11, 2019

DOI: $10.3892 /$ etm.2020.9275

\begin{abstract}
This study aimed to investigate the clinical efficacy of oxcarbazepine and lamotrigine combined with escitalopram in treating patients with epilepsy and depressive disorder, and their influence on the prognostic quality of life. A total of 108 patients with epilepsy and depression were selected as research participants. Among them, 53 patients were treated by oxcarbazepine combined with escitalopram (group A) and 55 patients were treated by lamotrigine combined with escitalopram (group B). Following six-month treatment, efficacy, epilepsy frequency and duration, Hamilton Depression Rating (HAMD) and Montgomery-Asberg Depression Rating (MADRS) scores, adverse reactions, improvement of electroencephalogram (EEG) epileptic discharge, quality of life, 1-year drug retention rate and withdrawal reasons of the two groups were compared. There was no remarkable difference in the total efficacy rate between both groups. The number and duration of epileptic seizures, improvement of EEG epileptic discharge and quality of life in the two groups significantly improved after treatment, with no marked difference. HAMD and MADRS scores of patients from group B were significantly lower after treatment compared with those of patients from group A. The incidence rate of adverse reactions in group B was dramatically lower compared with group A, and the 1-year drug retention rate of group B was dramatically higher compared with that in group A. Both oxcarbazepine and lamotrigine combined with escitalopram exhibited good efficacy in patients with epilepsy and depressive disorder, and they may effectively improve the prognostic quality of life of patients. Lamotrigine combined with escitalopram presented with a better antidepressant effect and safety, with higher patient tolerance.
\end{abstract}

Correspondence to: Dr Wenli Zhao, Department of Neurosurgery, Weifang No. 2 People's Hospital, 7 Yuanxiao Street, Kuiwen, Weifang, Shandong 261041, P.R. China

E-mail: enw4e2@163.com

Key words: oxcarbazepine, lamotrigine, escitalopram, epilepsy and depressive disorder comorbidity, comparison of clinical efficacy, quality of life

\section{Introduction}

Epilepsy is a common neurological encephalopathy mainly caused by abnormal discharge of brain neurons, with transient functional abnormalities of the nervous system as the main characteristic (1). Epileptic patients who suffer from long-term recurrent seizures are likely to develop further brain injury (2). Recently, the development of medical technology had made the diagnosis of epilepsy more accurate, and epilepsy treatment is mainly based on antiepileptic drugs. However, although the symptoms of epilepsy are manageable in most patients, not all can be effectively treated $(3,4)$.

Antiepileptic drugs comprise traditional and new antiepileptic drugs. Traditional antiepileptic drugs are mainly represented by carbamazepine. Although they have good efficacy, compared with new epilepsy drugs, most patients have more adverse reactions when taking traditional antiepileptic drugs, leading to drug intolerance, which causes patients to end treatment $(5,6)$. Oxcarbazepine, a copper-based derivative of carbamazepine, can reduce the induction of liver enzymes and improve patient tolerance while playing a therapeutic role (7). Lamotrigine is also an antiepileptic drug, which is widely used clinically. As a derivative of benzotriazine, lamotrigine not only has better efficacy in epilepsy, but also has less of an effect on liver and kidney function (8). The incidence of depression is high in epileptic patients due to the use of long-term medication and the adverse effects of the disease on quality of life. Depression will not only further aggravate the epileptic seizures, but also seriously affects the quality of life of patients (9). Therefore, it is imperative for epileptic patients to receive antidepressant treatment while treating epilepsy. However, both lamotrigine and oxcarbazepine have only recently been used in patients with epilepsy and depression, with relatively few clinical applications $(10,11)$. Escitalopram, a new antidepressant, can inhibit the reuptake of 5-hydroxytryptamine (5-HT) to achieve a better antidepressant effect (12).

However, to the best of our knowledge, relatively few studies on the efficacy of oxcarbazepine and lamotrigine combined with escitalopram in epileptic patients have been performed. Hence, the efficacy of oxcarbazepine and lamotrigine combined with escitalopram in epileptic patients and its influence on prognostic quality of life were studied, so 
as to provide a more appropriate treatment scheme for patients with epilepsy and depression.

\section{Materials and methods}

General data. A total of 108 patients with epilepsy combined with depression were selected for the present study between July 2014 to March 2017. There were 58 males and 50 females, with an age range of 28-38 years (mean age, 33.97 \pm 3.26 years). All were of Han Chinese descent. Loss of consciousness was the main seizure symptom. Among the cohort, 53 patients treated with oxcarbazepine combined with escitalopram were assigned to group A, and 55 patients treated with lamotrigine combined with escitalopram were assigned to group B. The inclusion criteria were as follows: i) Patients who met the criteria for diagnosis and classification of epilepsy with depression (11); ii) course of disease $>3$ months and seizure frequency $>1$ month; and iii) patients with depressive disorder (Zung Self-Rating Depression Scale >53) (11). The exclusion criteria were as follows: i) Patients had previously taken antiepileptic or antidepressant drugs; ii) patients with severe liver and kidney dysfunction; iii) pregnant or lactating patients; iv) patients with central nervous system diseases such as cerebral infarction and hemorrhage; v) patients with communication and cognitive dysfunction; and vi) patients who did not cooperate with the experiment. Patients agreed to participate in the experiment and signed written informed consent.

Treatment plan. Patients in group A were treated with oxcarbazepine (Novartis International AG) combined with escitalopram. Oxcarbazepine was initially taken at a dose of $300 \mathrm{mg} /$ day twice a day, and the dose was increased by $100 \mathrm{mg}$ every 3 days until the does reached $900 \mathrm{mg}$. On this basis, escitalopram (Sichuan Kelun Pharmaceutical Co., Ltd.) was added, and the initial dose was $5 \mathrm{mg} / \mathrm{d}$, once a day. After one week, the dose was adjusted according to patients' tolerance. The maximum dose was no more than $10 \mathrm{mg} / \mathrm{d}$.

Patients in group B were treated with lamotrigine (Guilin Sanjin Pharmaceutical Co., Ltd.) combined with escitalopram. The initial dose of lamotrigine was $25 \mathrm{mg} /$ day, twice a day. After 1-week administration, the daily dose was adjusted by increasing the dose by $25 \mathrm{mg}$ per week. Once the dose reached the effective therapeutic dose, the increase was stopped, with the maximum dose not exceeding $150 \mathrm{mg} /$ day. The initial dose of escitalopram was $5 \mathrm{mg} /$ day, once a day. After 1 week, the dose was adjusted according to the tolerance of patients, with the maximum dose not exceeding $10 \mathrm{mg} /$ day.

Observational indicators. The efficacy of the drugs after 6-month treatment in patients from the two groups was evaluated and categorized as 'cured' (no seizure during the observation period), 'significantly effective' (seizure frequency reduced by $>75 \%$ ), 'effective' (seizure frequency reduced by $>50 \%$ ) and 'ineffective' (seizure frequency not significantly reduced or even increased). The total efficacy rate of the treatment was calculated based on the following formula: (Number of 'cured' + number of 'significantly effective' + number of 'effective')/total number of patients x100. The frequency and duration of epileptic seizures were recorded and compared before and 6 months after treatment. Hamilton Depression Rating (HAMD) (13) and Montgomery-Asberg
Depression Rating (MADRS) (14) scores were used to evaluate and compare the depressive states of patients from both groups before and 6 months after treatment. The improvement of electroencephalogram (EEG) epileptic discharge between the two groups was categorized as 'control' (epileptic discharge completely disappeared), 'significantly improved' (epileptic discharge was reduced by $>50 \%$ ), 'improved' (epileptic discharge was reduced by $>25 \%$ ) and 'ineffective' (epileptic discharge was reduced by $<25 \%$ ). The improvement rate was calculated using the following formula: (Number of 'control' patients + number of 'significantly improved' patients + number of 'improved' patients)/total number of patients x100. Adverse reactions of patients, which included nausea, lethargy, dizziness, headache, rash and loss of appetite from both groups throughout 6-month treatment were recorded and compared. The Quality of Life in Epilepsy-Patients-Weighted 31p scoring system was used to evaluate and compare the quality of life of patients 6 months after treatment (15). The following domains were assessed: Emotion, cognition, social relations, energy, health status and overall quality of life, with a total score of 100 points. Higher scores represent a higher quality of life. The patients were followed up by telephones, outpatient services and WeChat (Tencent) for 1 year. The 1-year drug retention rates of patients from the two groups were recorded and compared, and the time and reason of drug withdrawal were recorded.

Statistical analysis. SPSS 19.0 (IBM Corp.) was used for statistical analysis and data were expressed as the mean \pm SD $(n=3)$. Counting data were analyzed using $\chi^{2}$ test. Comparisons between two groups were analyzed by Student's unpaired t-test and those before and after treatment within one group were analyzed by paired t-test. Two-way ANOVA was used for multiple comparisons, followed by Tukey's HSD post hoc test. GraphPad Prism 6.0 (GraphPad, Inc.) was used for graphical representation. $\mathrm{P}<0.05$ was considered to be statistically significant.

\section{Results}

Comparison of general data. No significant difference in sex, age, body mass index, course of disease, marital status, seizure type, creatinine and urea nitrogen levels and place of residence was found between the two groups (Table I).

Comparison of drug efficacy in patients. No significant difference in the total efficacy rate of treatment was found between the two groups (Table II).

Comparison of frequency and duration of epileptic seizures before and after treatment. The epileptic seizure frequency of patients from group A before and 6 months after treatment was $3.21 \pm 0.42$ times/month and $1.28 \pm 0.25$ times/month, with a duration of $5.21 \pm 1.26$ and $3.03 \pm 0.54 \mathrm{~min}$, respectively. The frequency of epileptic seizures of patients from group B before and six months after treatment was $3.18 \pm 0.39$ times/month and $1.19 \pm 0.26$ times/month, with a duration of $5.28 \pm 1.31$ and $3.07 \pm 0.50 \mathrm{~min}$, respectively. The frequency and duration of epileptic seizures after treatment in both groups decreased significantly compared with before treatment. No significant difference was found in the frequency and duration of epileptic seizures between groups A and $\mathrm{B}$ before or after treatment (Fig. 1). 
A



B

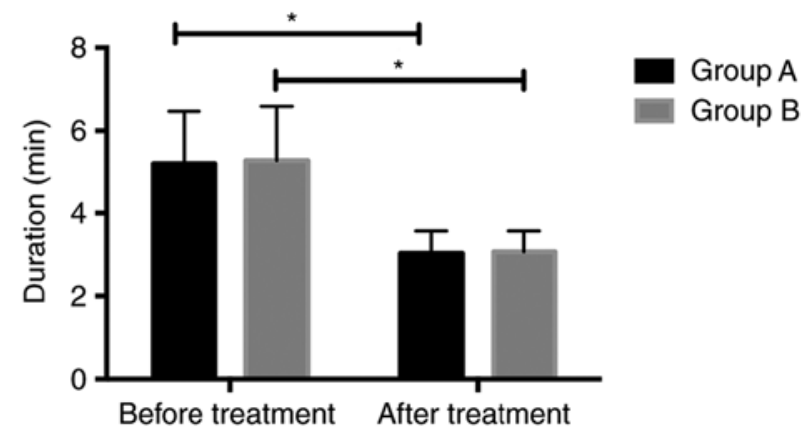

Figure 1. Comparison of frequency and duration of epileptic seizures of patients before and after treatment. (A) The frequency of epileptic seizures after treatment in groups A and B significantly decreased compared with before treatment. (B) The duration of epileptic seizures after treatment in groups A and B was significantly lower compared with before treatment. ${ }^{*} \mathrm{P}<0.05$.
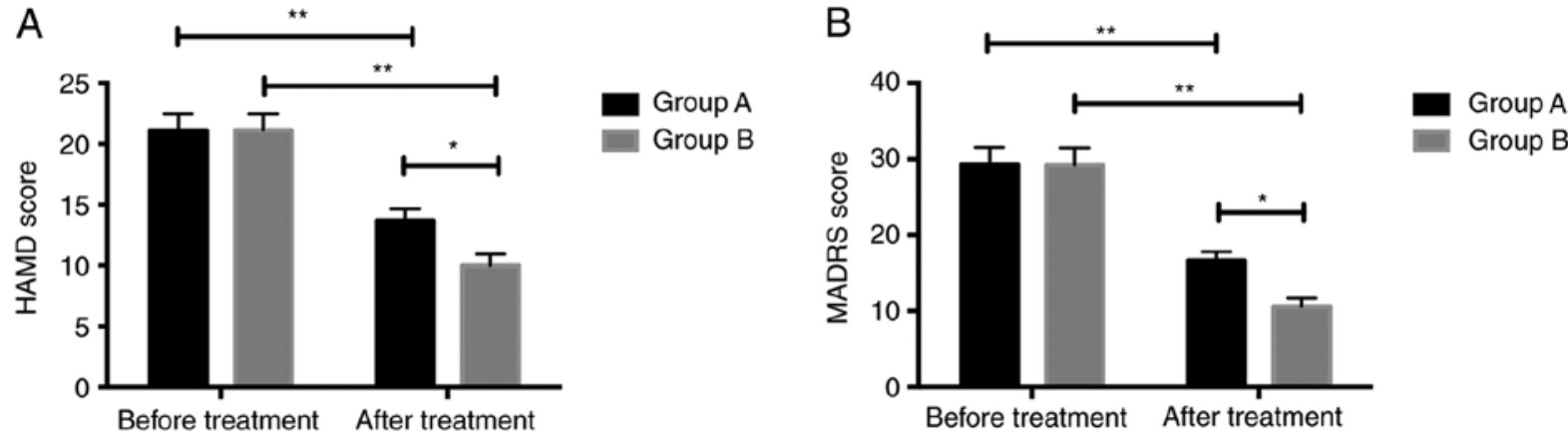

Figure 2. Comparison of HAMD and MADRS scores of patients before and after treatment. (A) The HAMD scores of patients in groups A and B after treatment were significantly lower compared with before treatment. The HAMD scores of patients of group B after treatment were significantly lower compared with those of patients from group A. (B) The MADRS score of patients from groups A and B after treatment were significantly lower compared with those before treatment. The MADRS scores of patients from group B after treatment were significantly lower compared with those of patients from group A. $\mathrm{P}<0.05$ and ${ }^{* *} \mathrm{P}<0.001$. HAMD, Hamilton Depression Rating; MADRS, Montgomery-Asberg Depression Rating.

Comparison of HAMD and MADRS scores before and after treatment. The HAMD scores of patients from group A were $21.11 \pm 1.37$ and $13.67 \pm 0.98$ before and six months after treatment, respectively, while the MADRS scores of group A patients were $29.33 \pm 2.21$ and $16.65 \pm 1.18$ before and six months after treatment, respectively. The HAMD scores of patients from group B were $21.13 \pm 1.35$ and $9.98 \pm 0.96$ before and six months after treatment, respectively, while the MADRS scores of group B patients were $29.22 \pm 2.25$ and $10.59 \pm 1.09$ before and six months after treatment, respectively. The HAMD and MADRS scores of patients of both groups after treatment were significantly lower compared before treatment. The scores of patients in group B after treatment were significantly lower compared with those of group A patients (Fig. 2).

Improvement of EEG epileptic discharge of patients in the two groups. No significant difference in the improvement rate of EEG epileptic discharge was measured between the two groups $(\mathrm{P}>0.05)$. The control rate of group $\mathrm{B}$ was significantly higher than that of group $\mathrm{A}(\mathrm{P}=0.001)$ (Table III).

Comparison of adverse reactions from patients. The total incidence rate of adverse reactions of patients in group B was significantly lower compared with that of those in group A (Table IV).
Comparison of quality of life of patients before and after treatment. Emotional, cognitive, social relationship, energy, health status and overall quality of life scores before treatment in group A were 43.92 $\pm 11.37,47.79 \pm 12.18,42.96 \pm 13.62$, $43.09 \pm 11.79$ and $41.67 \pm 14.66$, respectively. Emotional, cognitive, social relationship, energy, health status and overall quality of life scores after treatment in group A were 58.74 \pm 13.54 , $57.34 \pm 14.23,57.29 \pm 18.63,56.31 \pm 15.58$ and $62.92 \pm 17.54$, respectively. Emotional, cognitive, social relationship, energy, health status and overall quality of life scores before treatment in group B were $43.67 \pm 11.26,48.01 \pm 12.21,43.11 \pm 12.99$, $43.17 \pm 11.67$ and $41.89 \pm 13.91$, respectively. Emotional, cognitive, social relationship, energy, health status and overall quality of life scores after treatment in group B were 58.71 $\pm 13.41,57.26$ $\pm 14.31,57.33 \pm 18.14,56.27 \pm 15.43$ and $63.56 \pm 17.33$, respectively. No significant difference in the quality of life was measured between both groups before treatment. Quality of life scores in the two groups were all higher after treatment compared with before treatment. No significant difference in the quality of life after treatment was found between groups A and B (Fig. 3).

One-year drug withdrawal rate and withdrawal reason records of patients. Twenty patients in group A stopped taking the drugs within one year, with a withdrawal rate of $37.73 \%$. Among them, 4 patients stopped taking the drugs due to poor efficacy, 
Table I. General characteristics of patients.

\begin{tabular}{|c|c|c|c|c|}
\hline Characteristics & $\begin{array}{c}\text { Group A } \\
n=53\end{array}$ & $\begin{array}{c}\text { Group B } \\
n=55\end{array}$ & $\mathrm{t} / \chi^{2}$ value & P-value \\
\hline Sex & & & 0.032 & 0.858 \\
\hline Male & $28(52.83)$ & $30(54.55)$ & & \\
\hline Female & $25(47.17)$ & $25(45.45)$ & & \\
\hline Age, years & & & 0.157 & 0.692 \\
\hline$\leq 33$ & $24(45.28)$ & $27(49.10)$ & & \\
\hline$>33$ & $29(54.72)$ & $28(50.90)$ & & \\
\hline $\mathrm{BMI}, \mathrm{kg} / \mathrm{m}^{2}$ & & & 0.000 & 0.997 \\
\hline$\leq 22$ & $26(49.06)$ & $27(49.10)$ & & \\
\hline$>22$ & $27(50.94)$ & $28(50.90)$ & & \\
\hline Course of disease, years & $3.52 \pm 0.26$ & $3.49 \pm 0.24$ & 0.623 & 0.534 \\
\hline Marital status & & & 0.019 & 0.892 \\
\hline Married & $35(66.04)$ & 37 (67.27) & & \\
\hline Unmarried & $18(33.96)$ & $18(32.73)$ & & \\
\hline Seizure type & & & 0.125 & 0.724 \\
\hline Systemic & $31(58.49)$ & $34(61.82)$ & & \\
\hline Partial & $22(41.51)$ & $21(38.18)$ & & \\
\hline Creatinine, $\mu \mathrm{mol} / 1$ & $69.31 \pm 9.26$ & $70.09 \pm 9.37$ & 0.435 & 0.665 \\
\hline Urea nitrogen, mmol/1 & $6.15 \pm 1.42$ & $6.11 \pm 1.37$ & 1.049 & 0.882 \\
\hline Place of residence & & & 0.040 & 0.842 \\
\hline Cities and towns & $28(52.83)$ & $28(50.91)$ & & \\
\hline Countryside & $25(47.17)$ & 27 (49.09) & & \\
\hline
\end{tabular}

Data are expressed as the mean \pm SD or number (percentage). BMI, body mass index.

Table II. Comparison of drug efficacy between patients groups.

\begin{tabular}{|c|c|c|c|c|}
\hline Efficacy & $\begin{array}{c}\text { Group A } \\
n=53\end{array}$ & $\begin{array}{c}\text { Group B } \\
n=55\end{array}$ & $\mathrm{t} / \chi^{2}$ value & P-value \\
\hline Cured & $25(47.17)$ & $26(47.27)$ & 0.000 & 0.992 \\
\hline Significantly effective & $16(30.19)$ & $17(30.91)$ & 0.007 & 0.935 \\
\hline Effective & $6(11.32)$ & $7(12.73)$ & 0.050 & 0.822 \\
\hline Ineffective & $6(11.32)$ & $5(9.09)$ & 0.147 & 0.702 \\
\hline Total efficacy rate & $47(88.68)$ & $50(90.90)$ & 0.147 & 0.702 \\
\hline
\end{tabular}

Data are expressed as number (percentage).

Table III. Improvement of electroencephalogram epileptic discharge in patients

\begin{tabular}{|c|c|c|c|c|}
\hline Efficacy & $\begin{array}{c}\text { Group A } \\
n=53\end{array}$ & $\begin{array}{c}\text { Group B } \\
\mathrm{n}=55\end{array}$ & $\mathrm{t} / \chi^{2}$ value & $\mathrm{P}$-value \\
\hline Control & $11(20.75)$ & $23(41.82)$ & 10.89 & 0.001 \\
\hline Significantly improved & $21(39.62)$ & $12(21.82)$ & 4.032 & 0.045 \\
\hline Improved & $14(26.42)$ & $12(21.82)$ & 0.312 & 0.576 \\
\hline Ineffective & $7(13.21)$ & $8(14.55)$ & 0.040 & 0.841 \\
\hline Improvement rate & $46(86.79)$ & $47(85.45)$ & 0.040 & 0.841 \\
\hline
\end{tabular}

Data are expressed as number (percentage). 
Table IV. Comparison of adverse reactions between patients groups.

\begin{tabular}{lcccr}
\hline Reaction & $\begin{array}{c}\text { Group A } \\
\mathrm{n}=53\end{array}$ & $\begin{array}{c}\text { Group B } \\
\mathrm{n}=55\end{array}$ & $\mathrm{t} / \chi^{2}$ value & P-value \\
\hline Nausea & $1(5.66)$ & 0 & 1.047 & 0.306 \\
Sleepiness & $2(3.77)$ & $1(1.82)$ & 0.382 & 0.567 \\
Dizziness, headache & $2(3.77)$ & $1(1.82)$ & 0.382 & 0.567 \\
Rash & $6(3.77)$ & $1(1.82)$ & 4.021 & 0.045 \\
Loss of appetite & $1(5.66)$ & $1(1.82)$ & 0.000 & 0.979 \\
Overall incidence rate of adverse reactions & $12(22.64)$ & $4(7.27)$ & 5.052 & 0.025 \\
\hline
\end{tabular}

Data are expressed as number (percentage).

A

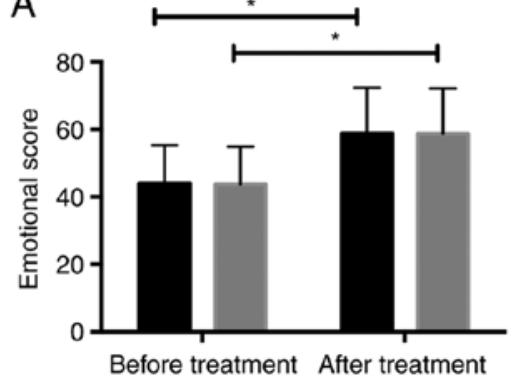

C

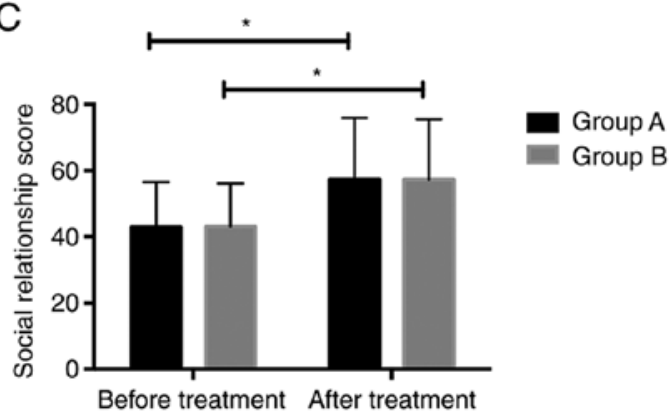

Group A

Group B
B

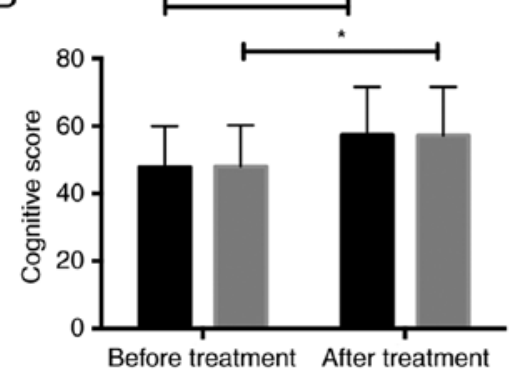

D

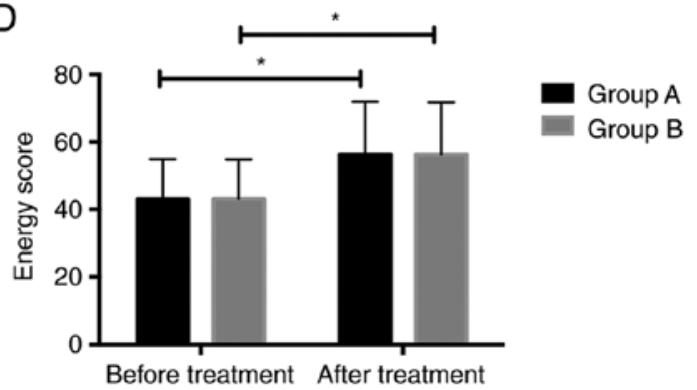

Group A

Group B
E

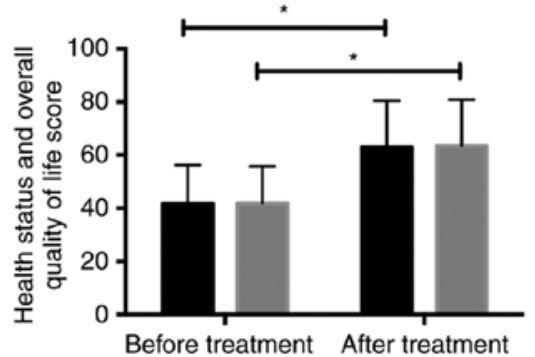

Group A

Group B
0.306

0.567

0.045

0.025 
Table V. One-year drug withdrawal rate of patients.

\begin{tabular}{lcccr}
\hline Factor & $\begin{array}{c}\text { Group A } \\
\mathrm{n}=53\end{array}$ & $\begin{array}{c}\text { Group B } \\
\mathrm{n}=55\end{array}$ & $\mathrm{t} / \chi^{2}$ value & P-value \\
\hline Drug withdrawal & & & 5.144 & 0.023 \\
Yes & $20(37.73)$ & $10(18.18)$ & & \\
No & $33(62.26)$ & $45(81.82)$ & & \\
\hline
\end{tabular}

Data are expressed as number (percentage).

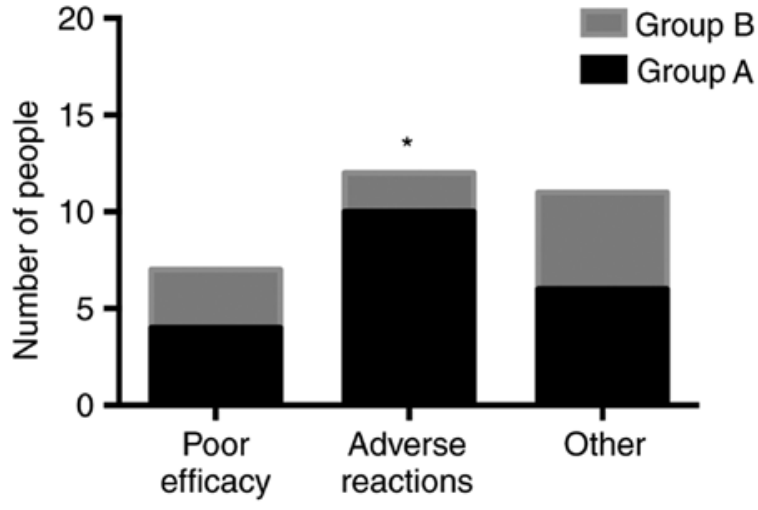

Figure 4. Reasons for patient drug withdrawal. The number of patients in group A who stopped taking drugs due to adverse reactions was significantly higher compared with that in in group $\mathrm{B}$. ${ }^{*} \mathrm{P}<0.05$.

of brain neurons. Its pathogenesis is very complex and it is often accompanied by depressive disorders (16). Epilepsy and depressive disorder are pathogenically similar and might be caused by reduced neurotransmitter release (17). The occurrence of epilepsy could promote the production of excitatory amino acids. This could lead to a reduction in 5-HT activity, which is one of the main causes of depression (18). A decrease in 5-HT activity can also further induce epilepsy, resulting in a cycle of epilepsy and depressive disorder (19).

The present study explored the efficacy of two new antiepileptic drugs (oxcarbazepine and lamotrigine) combined with the antidepressant escitalopram for the treatment of epilepsy combined with depressive disorder. The efficacy in patients from the two groups was compared, and the results showed that there was no significant difference in the total efficacy rates. The frequency and duration of epileptic seizures after treatment from the two groups significantly reduced compared with before treatment, and EEG epileptic discharge also improved. These results suggested that oxcarbazepine and lamotrigine had good efficacy in epilepsy patients. Another study showed that oxcarbazepine and lamotrigine had good efficacy on epilepsy patients, consistent with the present results (20). The main mechanism of oxcarbazepine is inhibiting the repeated discharge of neurons by blocking voltage-dependent sodium ion channels in brain cells (21). The main mechanism of lamotrigine is inhibiting voltage-dependent calcium and sodium channels to control the temperature of the presynaptic membrane and inhibit the release of neurotransmitters, ultimately reducing abnormal discharge of neurons (22). The HAMD and MADRS scores of patients from both groups before and after treatment were also compared. The results showed that the two scores of patients after treatment were significantly lower compared with before treatment and the scores of patients in group B after treatment were obviously lower compared those of patients in group A. The results suggested that the depressive disorders in patients from the two groups after treatment were significantly relieved, and lamotrigine combined with escitalopram was more effective than oxcarbazepine combined with escitalopram at relieving depressive disorders. A previous study verified that lamotrigine showed antiepileptic and anti-bipolar depression effects (23). Lamotrigine treatment managed epileptic symptoms, and improved depressive symptoms and the quality of life of patients (24). Escitalopram was chosen as a combination drug as it has a selective inhibitory effect on 5-HT and an antidepressant effect (25).

Subsequently, the adverse reactions of patients from the two groups were recorded and compared. The results showed that the total incidence rate of adverse reactions of patients from group B was significantly lower compared with that of group A. Although the incidence rate of rash in patients from group A was higher compared with that in group B patients, all patients experienced relief from their adverse reactions after symptomatic treatment. A previous study indicated that rash was the most common adverse reaction of oxcarbazepine, which was due to the possibility of rash induced by the human leukocyte antigen allele HLA-B*1502 (26). Finally, the quality of life before and after treatment and the 1-year drug retention rate of patients from both groups were compared. The results showed that the quality of life scores of patients after treatment significantly improved compared with before treatment. The 1-year drug retention rate of patients from group B was significantly higher compared with patients from group A. A previous study found that the drug retention rate of oxcarbazepine was significantly lower compared with that of lamotrigine when recording the drug retention rate of epileptic patients 3 years after treatment (27). Another study found that the main reason for the lower drug retention rate of oxcarbazepine compared with lamotrigine was the higher rate of adverse reactions, which was also the primary reason for patients to stop taking the drugs (28).

To summarize, oxcarbazepine and lamotrigine combined with escitalopram showed good efficacy in epileptic patients with depressive disorder, and they may effectively improve quality of life. Lamotrigine combined with escitalopram presented with a better antidepressant effect and better safety compared with oxcarbazepine and escitalopram. However, there are still some limitations to the present study. For example, the efficacy of different doses of drugs has not been discussed. There may be some differences in the conclusion due to the 
small sample size; on the other hand, because of individual differences, the dosages of drugs were not the same among all patients, which may also lead to some differences in the results.

\section{Acknowledgements}

Not applicable.

\section{Funding}

No funding was received.

\section{Availability of data and materials}

The datasets used and/or analyzed during the current study are available from the corresponding author on reasonable request.

\section{Authors' contributions}

$\mathrm{XZ}$ conceived and designed the study and drafted the paper. $\mathrm{XZ}$ and WZ collected, analyzed and interpreted the experimental data, and critically revised the manuscript. Both authors read and approved the final manuscript.

\section{Ethics approval and consent to participate}

The study was approved by the Ethics Committee of Sunshine Union Hospital. Written informed consent was obtained from patients.

\section{Patient consent for publication}

Not applicable.

\section{Competing interests}

The authors declare that they have no competing interests.

\section{References}

1. Kanner AM, Ashman E, Gloss D, Harden C, Bourgeois B, Bautista JF, Abou-Khalil B, Burakgazi-Dalkilic E, Llanas Park E, Stern J, et al: Practice guideline update summary: Efficacy and tolerability of the new antiepileptic drugs II: Treatment-resistant epilepsy: Report of the Guideline Development, Dissemination, and Implementation Subcommittee of the American Academy of Neurology and the American Epilepsy Society. Neurology 92: 82-90, 2018.

2. Chen Z, Brodie MJ, Liew D and Kwan P: Treatment outcomes in patients with newly diagnosed epilepsy treated with established and new antiepileptic drugs: A 30-year longitudinal cohort study. JAMA Neurol 75: 279-286, 2018.

3. Cooper YA, Pianka ST, Alotaibi NM, Babayan D, Salavati B, Weil AG, Ibrahim GM, Wang AC and Fallah A: Repetitive transcranial magnetic stimulation for the treatment of drug-resistant epilepsy: A systematic review and individual participant data meta-analysis of real-world evidence. Epilepsia Open 3: 55-65, 2017.

4. Beamer E, Fischer W and Engel T: The ATP-gated P2X7 receptor as a target for the treatment of drug-resistant epilepsy. Front Neurosci 11: 21, 2017.

5. Zhang L, Wang $\mathrm{C}$ and Li W: A meta-analysis of randomized controlled trials on levetiracetam in the treatment of pediatric patients with epilepsy. Neuropsychiatr Dis Treat 14: 769-779, 2018

6. Liu JT, Liu B and Zhang H: Surgical versus medical treatment of drug-resistant epilepsy: A systematic review and meta-analysis. Epilepsy Behav 82: 179-188, 2018.
7. Yun JH: Comparison of lamotrigine and oxcarbazepine monotherapy for pediatric focal epilepsy: An observational study. Seizure 60: 123-126, 2018.

8. Tombini M, Pellegrino G, Assenza G and Lazzaro VD: De novo multifocal myoclonus induced by lamotrigine in a temporal lobe epilepsy case. J Neurol Sci 373: 31-32, 2017.

9. Singh T, Kaur T and Goel RK: Adjuvant quercetin therapy for combined treatment of epilepsy and comorbid depression. Neurochem Int 104: 27-33, 2017.

10. Luszczki JJ, Wlaz A, Karwan S, Florek-Luszczki M and Czuczwar SJ: Effects of WIN 55,212-2 mesylate on the anticonvulsant action of lamotrigine, oxcarbazepine, pregabalin and topiramate against maximal electroshock-induced seizures in mice. Eur J Pharmacol 720: 247-254, 2013.

11. Thelengana A, Shukla G, Srivastava A, Singh MB, Gupta A, Rajan R, Vibha D, Pandit AK and Prasad K: Cognitive, behavioural and sleep-related adverse effects on introduction of levetiracetam versus oxcarbazepine for epilepsy. Epilepsy Res 150: 58-65, 2019.

12. Gorman JM, Korotzer A and Su G: Efficacy comparison of escitalopram and citalopram in the treatment of major depressive disorder: Pooled analysis of placebo-controlled trials. CNS Spectr 7 (Suppl 1): 40-44, 2002.

13. Gill SJ, Lukmanji S, Fiest KM, Patten SB, Wiebe S and Jetté N: Depression screening tools in persons with epilepsy: A systematic review of validated tools. Epilepsia 58: 695-705, 2017.

14. Carneiro AM, Fernandes F and Moreno RA: Hamilton depression rating scale and montgomery-asberg depression rating scale in depressed and bipolar I patients: psychometric properties in a Brazilian sample. Health Qual Life Outcomes 13: 42, 2015.

15. Wijnen BF, Mosweu I, Majoie MH, Ridsdale L, de Kinderen RJ, Evers SM and McCrone P: A comparison of the responsiveness of EQ-5D-5L and the QOLIE-31P and mapping of QOLIE-31P to EQ-5D-5L in epilepsy. Eur J Health Econ 19: 861-870, 2018.

16. Peng WF, Fan F, Li X, Zhang QQ, Ding J and Wang X: Different behavioral and pathological changes between epilepsy-associated depression and primary depression models. Epilepsy Behav 83: 212-218, 2018.

17. Rider FK, Danilenko OA, Grishkina MN, Kustov GV, Akzhigitov RG, Lebedeva AV and Gekht AB: Depression and epilepsy: Comorbidity, pathogenetic similarity, and principles of treatment. Neurosci Behav Physiol 48: 78-82, 2018.

18. Josephson CB, Lowerison M, Vallerand I, Sajobi TT, Patten S, Jette $\mathrm{N}$ and Wiebe $\mathrm{S}$ : Association of depression and treated depression with epilepsy and seizure outcomes: A multicohort analysis. JAMA Neurol 74: 533-539, 2017.

19. Mula M: Depression in epilepsy. Curr Opin Neurol 30: 180-186, 2017.

20. Johannessen Landmark C, Baftiu A, Tysse I, Valsø B, Larsson PG, Rytter E and Johannessen SI: Pharmacokinetic variability of four newer antiepileptic drugs, lamotrigine, levetiracetam, oxcarbazepine, and topiramate: A comparison of the impact of age and comedication. Ther Drug Monit 34: 440-445, 2012.

21. Glauser TA: Oxcarbazepine in the treatment of epilepsy. Pharmacother J Hum Pharmacol Drug Ther 21: 904-919, 2012.

22. D'Anto J, Wnuk W, Rossetti AO, Decosterd LA, Buclin T and Novy J: Lamotrigine serum levels: Ceiling effect in people with epilepsy in remission? Epilepsy Behav 74: 41-44, 2017.

23. Zhang M, Ming LI, Zhang X, et al: The clinical efficacy of lamotrigine in the treatment of epilepsy with depression and the influence on cognitive function. Chinese Journal of Medicinal Guide, 2017.

24. Martinović Z, Buder N, Milovanović M and Velićković R: Antiepileptic, behavioral, and antidepressant effects of adjuvant lamotrigine therapy in drug-resistant epilepsy. Vojnosanit Pregl 61: 485-490, 2004.

25. Azorin JM, Llorca PM, Despiegel N and Verpillat P: Escitalopram is more effective than citalopram for the treatment of severe major depressive disorder. Encephale 30: 158-166, 2004.

26. Kutluay E, McCague K, D'Souza J and Beydoun A: Safety and tolerability of oxcarbazepine in elderly patients with epilepsy. Epilepsy Behav 4: 175-180, 2003.

27. Sunwoo JS, Park BS, Ahn SJ, Hwang S, Park CY, Jun JS, Kim DW, Lee ST, Jung KH, Park KI, et al: Three-year retention rates of levetiracetam, topiramate, and oxcarbazepine: A retrospective Hospital-based Study. Clin Neuropharmacol 40: 56-62, 2017.

28. Reilly C, Atkinson P, Das KB, Chin RF, Aylett SE, Burch V, Gillberg C, Scott RC and Neville BG: Neurobehavioral comorbidities in children with active epilepsy: A population-based study. Pediatrics 133: e1586-e1593, 2014. 